\title{
Sum-Frequency Generation in Chiral Liquids near Electronic Resonance
}

\author{
M. A. Belkin, S. H. Han, X. Wei,* and Y. R. Shen ${ }^{\dagger}$ \\ Department of Physics, University of California, Berkeley, California 94720 \\ and Materials Sciences Division, Lawrence Berkeley National Laboratory, Berkeley, California 94720
}

(Received 29 May 2001; published 24 August 2001)

\begin{abstract}
We demonstrate experimentally that visible-visible sum-frequency generation in the bulk of a chiral liquid is observable near electronic resonant transitions. Although the process is electric dipole allowed, it is rather weak because the orientational average over molecules effectively reduces the bulk chiral nonlinearity.
\end{abstract}

DOI: $10.1103 /$ PhysRevLett.87.113001

There is currently a revived interest in optical sumfrequency generation (SFG) in chiral liquids as it could provide an effective means to probe molecular chirality. Giordmaine first pointed out in 1965 that in a chiral liquid, because of its lack of inversion symmetry, SFG is electric dipole allowed and should be readily detectable [1]. Rentzepis et al. reported in the following year the first observation of visible-visible SFG in an arabinose solution [2]. Their experimental finding, however, was not supported by others. In a 1993 paper, Shkurinov et al. briefly mentioned that they had successfully repeated Rentzepis' experiment [3], but as suggested in a recent article by Fischer et al., their observed signal most likely came from a four-wave mixing process instead of SFG [4]. The latter group failed to detect SFG from arabinose solutions as well as pinene liquid. They concluded, with the help of theoretical estimates, that the chiral nonlinearity in their samples should be at least 2 orders of magnitude smaller than that reported by Rentzepis et al. even near resonance. In a recent work, however, we were able to use resonantly enhanced infrared-visible SFG to obtain vibrational spectra of chiral liquids [5].

Visible-visible SFG mainly results from the electronic response of a medium. We believe that in a chiral liquid it should be observable if the sum frequency is close to a molecular absorption band and the corresponding electronic wave functions are sufficiently delocalized. We choose 1,1'-Bi-2-naphthol (BN) as a possible candidate (see Fig. 1 for the chemical structure of BN). Hicks and
PACS numbers: 33.55.Ad, 42.65.Ky, 78.20.Ek

co-workers found that optically active second harmonic generation (SHG) around the electronic transitions of a $\mathrm{BN}$ surface monolayer can be readily measured [6]. One would expect that the same transitions could be probed by visible-visible SFG in a bulk BN solution, although, as we shall see later, the chiral nonlinearity suffers a strong reduction from orientational average over molecules in a bulk liquid.

This paper reports our successful observation of visiblevisible SFG from BN solutions. We show that while the chiral nonlinearity of $\mathrm{BN}$ in solutions is small, it is easily measurable. Resonance enhancement helps for the detection and yields the SFG spectra for the two lowest electronic transitions in $\mathrm{BN}$. We have also attempted to measure SFG from arabinose (chemical structure: $\mathrm{C}_{5} \mathrm{H}_{10} \mathrm{O}_{5}$ ) solutions, but found that its chiral nonlinearity is below our detection limit even near resonance. The chiral nonlinearity of $\mathrm{BN}$ is about 2 orders of magnitude larger than the values predicted by Fischer et al. from their $a b$ initio calculations for small chiral molecules [4]. The result suggests that the more delocalized electrons of a relatively big $\mathrm{BN}$ molecule can yield a stronger chiral optical response.

Consider SFG at frequency $\omega=\omega_{1}+\omega_{2}$ by input fields $\vec{E}_{1}\left(\omega_{1}\right)$ and $\vec{E}_{2}\left(\omega_{2}\right)$ in a medium. Both the surface and the bulk nonlinear polarizations, $\vec{P}_{S}(\omega)$ and $\vec{P}_{B}(\omega)$ [7], induced in the medium can, in general, contribute to the SFG. Each contribution can be expressed in the multipole series form [8]:

$$
\begin{aligned}
& \vec{P}^{(2)}=\vec{P}_{D}^{(2)}-i \vec{k} \cdot \overleftrightarrow{Q}^{(2)}-\frac{1}{\omega} \vec{k} \times \vec{M}^{(2)}+\cdots,
\end{aligned}
$$

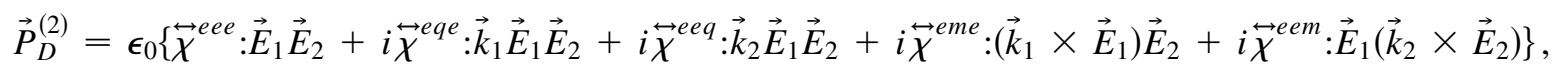

$$
\begin{aligned}
& \overleftrightarrow{Q}^{(2)}=\epsilon_{0} \overleftrightarrow{\chi}^{q e e}: \vec{E}_{1} \vec{E}_{2}, \quad \vec{M}^{(2)}=\omega \epsilon_{0} \stackrel{\leftrightarrow}{\chi}^{\text {mee }}: \vec{E}_{1} \vec{E}_{2}
\end{aligned}
$$

Here $\vec{P}_{D}^{(2)}, \overleftrightarrow{Q}^{(2)}$, and $\vec{M}^{(2)}$ represent the electric-dipole, electric-quadrupole, and magnetic-dipole polarizations, respectively, and $\chi$ 's are second-order nonlinear susceptibilities with superscripts $e, q$, and $m$ denoting electric-dipole, electric-quadrupole, and magnetic-dipole contributions, respectively, from the three fields involved in SFG.
For the bulk of an achiral liquid, $\stackrel{\leftrightarrow}{\chi}^{e e e}$ vanishes by symmetry, but $\stackrel{\leftrightarrow}{\chi}^{Q M}$ (we use $\overleftrightarrow{\chi}^{Q M}$ to represent, in general, the electric-quadrupole and magnetic-dipole terms: $\overleftrightarrow{\chi}^{e q e}, \overleftrightarrow{\chi}^{e m e}, \overleftrightarrow{\chi}^{q e e}$, etc.) can exist. At the surface, $\overleftrightarrow{\chi}^{e e e}$ no longer vanishes because of the broken inversion symmetry, and $\overleftrightarrow{\chi}^{Q M}$ is negligible. All the nonvanishing elements 


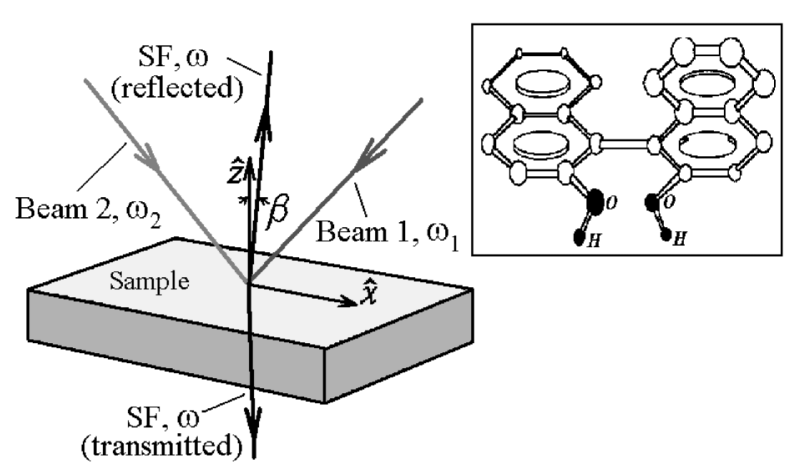

FIG. 1. Experimental arrangement and the structure of the BN molecule.

can in principle be measured by SFG using input/output beam polarization combinations $S S P$ (fields at $\omega, \omega_{1}$, and $\omega_{2}$ are $S, S$, and $P$ polarized, respectively), SPS, $P S S$, and $P P P$. For a chiral liquid, new nonvanishing elements pertaining to chirality appear in $\stackrel{\leftrightarrow}{\chi}^{e e e}$ for both the surface and the bulk [1]. The chiral elements in $\stackrel{\leftrightarrow}{\chi}^{Q M}$ are negligibly small at the surface and vanish by symmetry in the bulk. At the surface, the new chiral elements of $\overleftrightarrow{\chi}^{e e e}$ are $\chi_{x y z}=$ $-\chi_{y x z}, \chi_{y z x}=-\chi_{x z y}$, and $\chi_{z x y}=-\chi_{z y x}$ with $\hat{z}$ along the surface normal. In the bulk they are $\chi_{x y z}=-\chi_{y x z}=$ $\chi_{y z x}=-\chi_{x z y}=\chi_{z x y}=-\chi_{z y x}$, which we shall designate as $\chi_{\text {chiral }}$. These chiral elements can be accessed by SFG with polarization combinations $S P P, P S P$, and $P P S$.

The chiral nonlinear susceptibility $\chi_{\text {chiral }}$ can be related to the hyperpolarizabilities $\alpha_{\xi \eta \zeta}^{(2)}$ of the chiral molecules in the liquid:

$$
\begin{aligned}
\chi_{\text {chiral }}= & N l(\omega) l\left(\omega_{1}\right) l\left(\omega_{2}\right) \cdot \alpha_{\text {chiral }}, \\
\alpha_{\text {chiral }} \equiv\left\langle\alpha_{\xi \eta \zeta}\right\rangle=\frac{1}{6} & {\left[\alpha_{x^{\prime} y^{\prime} z^{\prime}}^{(2)}-\alpha_{y^{\prime} x^{\prime} z^{\prime}}^{(2)}+\alpha_{y^{\prime} z^{\prime} x^{\prime}}^{(2)}\right.} \\
& \left.\quad-\alpha_{z^{\prime} y^{\prime} x^{\prime}}^{(2)}+\alpha_{z^{\prime} x^{\prime} y^{\prime}}^{(2)}-\alpha_{x^{\prime} z^{\prime} y^{\prime}}^{(2)}\right],
\end{aligned}
$$

where $N$ is the density of molecules, the angular brackets denote an orientational average, $x^{\prime}, y^{\prime}$, and $z^{\prime}$ define the molecular axes, and $l(\Omega)=[\epsilon(\Omega)+2] / 3$ is the Lorentz local field correction factor. It can be shown that the microscopic expression of $\alpha_{\text {chiral }}$ takes the form

$$
\begin{aligned}
\alpha_{\text {chiral }}= & \frac{e^{3}}{6 \hbar^{2} \epsilon_{0}} \sum_{n} \frac{\left(\omega_{1}-\omega_{2}\right)}{\left(\omega-\omega_{n g}+i \Gamma_{n g}\right)} \\
& \times \sum_{n^{\prime}} \frac{\vec{r}_{g n} \cdot\left(\vec{r}_{n n^{\prime}} \times \vec{r}_{n^{\prime} g}\right)}{\left(\omega_{1}-\omega_{n^{\prime} g}\right)\left(\omega_{2}-\omega_{n^{\prime} g}\right)}
\end{aligned}
$$

assuming that $\omega$ is near electronic resonance and $\omega_{1}$ and $\omega_{2}$ are not. Here, $\omega_{i j}$ and $\Gamma_{i j}$ denote the transition frequency and damping constant for the transition between the states $|i\rangle$ and $|j\rangle$, and $\vec{r}_{i j}$ is the corresponding transition matrix element of $\vec{r}$. Comparing $\alpha_{\text {chiral }}$ in Eq. (3) with the expression of a single element of $\stackrel{\leftrightarrow}{\alpha}^{(2)}$, e.g., $\alpha_{x^{\prime} y^{\prime} z^{\prime}}^{(2)}[9]$ :

$$
\begin{aligned}
\alpha_{x^{\prime} y^{\prime} z^{\prime}}^{(2)}= & -\frac{e^{3}}{\hbar^{2} \epsilon_{0}} \sum_{n} \frac{x_{g n}^{\prime}}{\left(\omega-\omega_{n g}+i \Gamma_{n g}\right)} \\
& \times \sum_{n^{\prime}}\left[\frac{y_{n n^{\prime}}^{\prime} z_{n^{\prime} g}^{\prime}}{\omega_{1}-\omega_{n^{\prime} g}}+\frac{z_{n n^{\prime}}^{\prime} y_{n^{\prime} g}^{\prime}}{\omega_{2}-\omega_{n^{\prime} g}}\right],
\end{aligned}
$$

we notice that the value of $\alpha_{\text {chiral }}$ suffers a reduction from molecular orientational average through the factors $\left(\omega_{1}-\right.$ $\left.\omega_{2}\right)$ and $\left(\vec{r}_{g n} \times \vec{r}_{n n^{\prime}}\right) \cdot \vec{r}_{n^{\prime} g}$. As we shall see later, this is the reason why SFG from a chiral liquid is relatively weak although it is electric dipole allowed. In the special case of SHG with $\omega_{1}=\omega_{2}$, we have $\alpha_{\text {chiral }}=0$.

If we write $\vec{P}_{S}^{(2)}=\stackrel{\leftrightarrow}{\chi}(2): \vec{E}_{1} \vec{E}_{2}$ and $\vec{P}_{B}^{(2)}=\stackrel{\leftrightarrow}{\chi}_{B}^{(2)}: \vec{E}_{1} \vec{E}_{2}$, the SFG output takes the form [10]:

$$
\begin{aligned}
& S(\omega) \propto\left|\chi_{\text {eff }}^{(2)}\right|^{2}\left|\vec{E}_{1}\left(\omega_{1}\right)\right|^{2}\left|\vec{E}_{2}\left(\omega_{2}\right)\right|^{2}, \\
& \chi_{\mathrm{eff}}^{(2)}=[\hat{e} \cdot \overleftrightarrow{L}(\omega)] \cdot\left(\overleftrightarrow{\chi}_{S}^{(2)}+\frac{\overleftrightarrow{\chi}_{B}^{(2)}}{\left|\Delta k_{z}\right|}\right):\left[\hat{e}_{1} \cdot \overleftrightarrow{L}\left(\omega_{1}\right)\right] \\
& \times\left[\hat{e}_{2} \cdot \overleftrightarrow{L}\left(\omega_{2}\right)\right], \quad\left|\Delta k_{z}\right|=\left|k_{z}-k_{1 z}-k_{2 z}\right|,
\end{aligned}
$$

where $S(\omega)$ is the signal strength, $\hat{e}_{i}$ refers to the polarization of beam $i, \overleftrightarrow{L}(\Omega)$ is the Fresnel transmission coefficient at $\Omega$ at the interface, $l_{\text {coh }} \equiv 1 /\left|\Delta k_{z}\right|$ is the coherent length of interaction, and $\hat{z}$ is along the surface normal.

Our experimental arrangement, depicted schematically in Fig. 1, was similar to the one described elsewhere [7] except that both input beams were in the visible or near IR. They were generated by a mode-locked Nd:YAG/ optical parametric system with a pulse width of about $18 \mathrm{ps}$ and a repetition rate of $20 \mathrm{~Hz}$. Both beams were tunable in the wavelength range between $450 \mathrm{~nm}$ and $1.7 \mu \mathrm{m}$ with energy larger than $80 \mu \mathrm{J}$ per pulse. The SF outputs in transmission and reflection directions determined by matching of wave vector components along the sample surface, $k_{1 x}+k_{2 x}=k_{x}$, were measured by a gated photon counting system. The samples used in our experiment were $0.55 \mathrm{M}$ solutions of $\mathrm{BN}$ in tetrahydrofuran (THF) contained in a $2 \mathrm{~mm}$ thick fused quartz cell. In order to access $\chi_{\text {chiral }}$, the two input beams must be noncollinear. We set the input angles of the two beams at $44^{\circ}$ and $-34^{\circ}$, respectively. This led to a coherent length, $l_{\mathrm{coh}}=1 /\left|\Delta k_{z}\right|$, of about $300 \mathrm{~nm}$ for SFG in transmission and about $20 \mathrm{~nm}$ in reflection. The absorption length of the $\mathrm{BN}$ solution at the sum frequency $\omega$ was about $2 \mu \mathrm{m}$. To avoid the effect of absorption in transmitted SFG, the input beams were focused and overlapped to a $0.25 \mathrm{~mm}^{2}$ spot at the exit surface of the sample. For reflected SFG, they were overlapped at the entrance surface.

With $\omega$ in the absorption region of $\mathrm{BN}$, we observed rather strong SFG signals in transmission, but not in reflection, with the $S P P$ and $P P S$ polarization combinations. We did not detect any signal above the noise in achiral $P P P$ and SPS polarization combinations for both reflection and transmission. These results indicate that the bulk $\chi_{\text {chiral }}$ for the $\mathrm{BN}$ solution is detectable, and the bulk elements 
of $\stackrel{\leftrightarrow}{\chi}^{Q M}$ are much smaller than $\chi_{\text {chiral }}$; the surface chiral elements of $\leftrightarrow_{S}^{(2)}$ are also below our detection limit.

We present in Figs. 2(a) and 2(b) the SFG electronic spectra obtained in transmission with the SPP and PPS polarization combinations for the solutions of two enantiomers of $\mathrm{BN}$ and their racemic mixture in THF. They were taken with $\omega_{1}$ fixed at $9400 \mathrm{~cm}^{-1}(1.064 \mu \mathrm{m})$ and $\omega_{2}$ tunable from 19600 to $22200 \mathrm{~cm}^{-1}$ (510 to $450 \mathrm{~nm}$ ). As expected, SFG is absent in the racemic mixture and the spectra are identical for the two enantiomers because the SF signal is proportional to $\left|\chi_{\text {chiral }}\right|^{2}$. Normalization against SFG from a reference crystalline quartz plate allows us to deduce with the help of Eqs. (1) and (5) the value of $\left|\chi_{\text {chiral }}\right|^{2}$ versus $\omega$ (or wavelength $\lambda$ ). This is shown in Fig. 2(c) together with the absorption spectrum of the BN solution. It is then clear that the two peaks in the SFG spectra arise from resonance enhancement at the electronic transitions of BN. They can be identified with the lowest electronic transition $\left(S_{0} \rightarrow S_{1}\right)$ of BN that experiences excitonic splitting of the otherwise degenerate transitions in the separated 2-naphthol monomers [11].

To be sure that the observed chiral signal came from SFG in the bulk of the chiral BN solution, we measured its dependence on the two input beam powers. Presented in Figs. 3(a) and 3(b) are the results obtained with $\omega_{1}=$ $9400 \mathrm{~cm}^{-1}$ and $\omega_{2}=21700 \mathrm{~cm}^{-1}$ using the $S P P$ polarization combination. The observed dependence is linear, as expected from Eq. (5). We also measured with the same input frequencies and polarization combination the dependence of the signal on the $\mathrm{BN}$ concentration in the solution. Assuming the refractive indices of the solution hardly change with the BN concentration, $N$, Eqs. (2) and (5) pre-

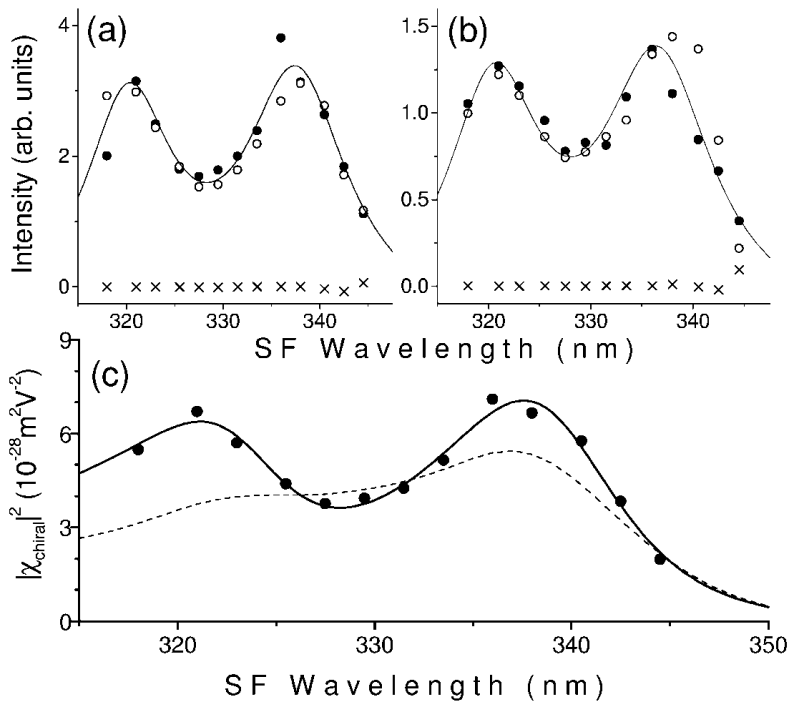

FIG. 2. Transmission SFG spectra of BN in THF with (a) SPP and (b) $P P S$ polarization combinations. Filled circles are for $S$-BN, open circles for $R$-BN, and crosses for the racemic mixture. Solid lines are fits to the data points. (c) Spectral dependence of $\left|\chi_{\text {chiral }}\right|^{2}$ in comparison with the absorption spectrum (dotted line). dict that the signal should be proportional to $N^{2}$. Indeed this is what we found as shown in Fig. 3(c). The data point at high $\mathrm{BN}$ concentration appears to deviate from the quadratic dependence presumably because the refractive indices and absorption length have changed appreciably. From Eq. (3), we also expect $\left|\chi_{\text {chiral }}\right|^{2} \propto\left(\omega_{1}-\omega_{2}\right)^{2}$, assuming the dependence on $\omega_{1}$ and $\omega_{2}$ in the denominator of Eq. (3) is negligible. To check this experimentally, we kept the sum frequency $\omega$ fixed at $30700 \mathrm{~cm}^{-1}(325 \mathrm{~nm})$ in our measurement, and tuned $\omega_{1}$ and $\omega_{2}$ simultaneously to vary $\left(\omega_{1}-\omega_{2}\right)$. Figure 4 shows that the measured $\left|\chi_{\text {chiral }}\right|^{2}$ versus $\left(\omega_{1}-\omega_{2}\right)$ does follow the quadratic dependence within the experimental uncertainty.

The experimental results described here clearly indicate that we have observed chiral SFG from enantiomeric $\mathrm{BN}$ solutions. The maximum measured value of $\left|\chi_{\text {chiral }}\right| / N \approx 9 \times 10^{-41} \frac{\mathrm{m}^{4}}{\mathrm{~V}}$ is however 2 orders of magnitude smaller than that of a typical element of surface chiral nonlinear susceptibility $\left|\left(\chi_{S}\right)_{i j k}\right| / N_{S} \approx 6 \times 10^{-39} \frac{\mathrm{m}^{4}}{\mathrm{~V}}$ for a BN monolayer of surface density $N_{S}$ on water. The latter quantity was obtained from an SHG measurement with $2 \omega$ in the range of $29000-34500 \mathrm{~cm}^{-1}$ and a $P$-in and $S$-out polarization combination. The relatively weak $\left|\chi_{\text {chiral }}\right| / N$ can be understood by comparing $\left|\chi_{\text {chiral }}\right| / N$ and $\left|\left(\chi_{S}\right)_{i j k}\right| / N_{S}$ in terms of the chiral molecular hyperpolarizability elements $\alpha_{i j k}^{(2)}(i \neq j \neq k)$. The surface SFG (or SHG) with the SPP polarization combination measures the quantity $\left(\chi_{S}\right)_{y x z}+\left(\chi_{S}\right)_{y z x}$. If the chiral molecules are well polar oriented at the surface, this measured quantity should have contributions mainly from a few selected elements of $\alpha_{i j k}^{(2)}$. For example, consider the case where the surface chiral molecules are oriented with their $z^{\prime}$

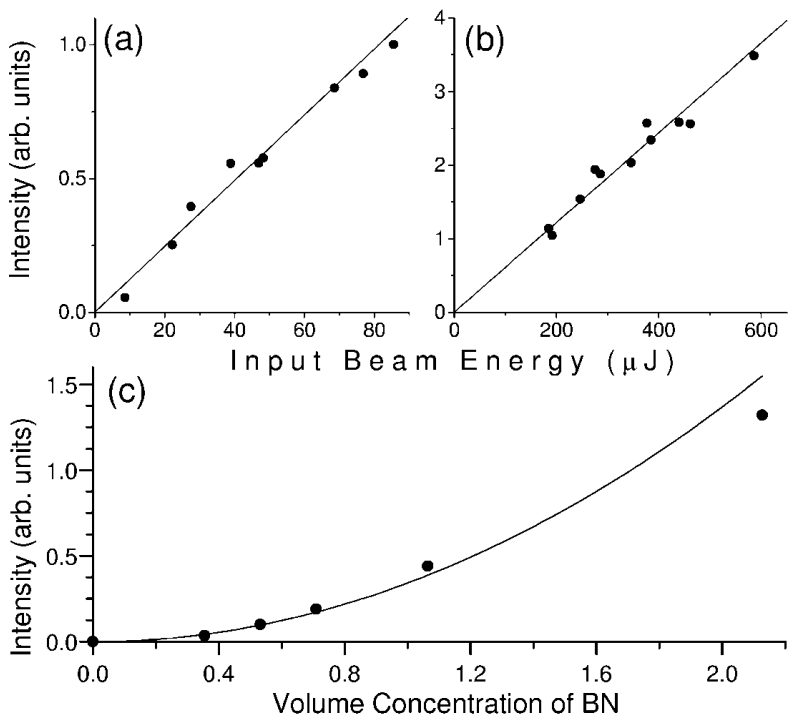

FIG. 3. Dependence of the SFG on the input power of (a) the beam at $21700 \mathrm{~cm}^{-1}$ and (b) the beam at $9400 \mathrm{~cm}^{-1}$. (c) Dependence of SFG on the BN concentration in solution (in terms of fractions of $0.55 \mathrm{M}$ solution). The solid lines are fits to the data points. 


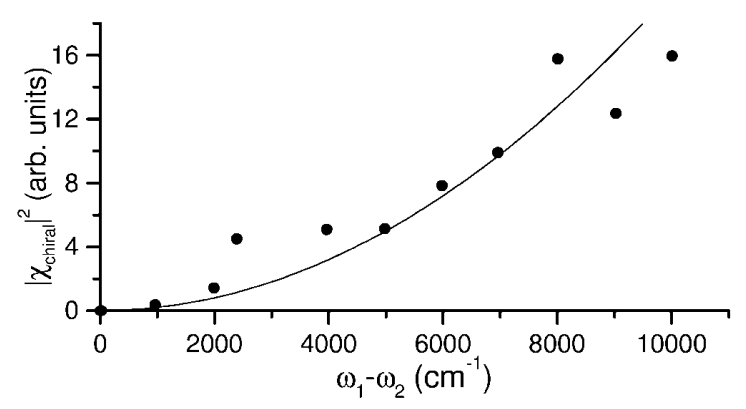

FIG. 4. Dependence of $\left|\chi_{\text {chiral }}\right|^{2}$ on $\left(\omega_{1}-\omega_{2}\right)$. The solid line is a fit to the data points.

axis parallel to the surface normal $\hat{z}$ and the $x^{\prime}$ and $y^{\prime}$ axes random in the surface plane. We find (neglecting the microscopic local field correction here)

$$
\frac{\left(\chi_{S}\right)_{y x z}+\left(\chi_{S}\right)_{y z x}}{N_{S}}=\frac{\alpha_{y^{\prime} x^{\prime} z^{\prime}}^{(2)}-\alpha_{x^{\prime} y^{\prime} z^{\prime}}^{(2)}+\alpha_{y^{\prime} z^{\prime} x^{\prime}}^{(2)}-\alpha_{x^{\prime} z^{\prime} y^{\prime}}^{(2)}}{2} .
$$

On the other hand, we have $\left|\chi_{\text {chiral }}\right| / N=\alpha_{\text {chiral }}$ with $\alpha_{\text {chiral }}$ given by Eq. (2). If the dispersion of $\stackrel{\leftrightarrow}{\alpha}^{(2)}$ with respect to $\omega_{1}$ and $\omega_{2}$ is neglected, we would have $\alpha_{i j k}^{(2)}=$ $\alpha_{i k j}^{(2)}$, and hence $\left[\left(\chi_{S}\right)_{y x z}+\left(\chi_{S}\right)_{y z x}\right] / N_{S}=\alpha_{y^{\prime} x^{\prime} z^{\prime}}^{(2)}-\alpha_{x^{\prime} y^{\prime} z^{\prime}}^{(2)}$ (i.e., nonzero in general), but from Eq. (2), $\chi_{\text {chiral }}=0$ even with $\omega_{1} \neq \omega_{2}$.

More explicitly, we notice from the microscopic expression of $\alpha_{\text {chiral }}$ in Eq. (3) that when $\left(\omega_{1}-\omega_{2}\right)$ is appreciable compared to $\omega_{1}$ or $\omega_{2}$, the main reduction factor for $\alpha_{\text {chiral }}$ comes from the quantity $\left(\vec{r}_{g n} \times \vec{r}_{n n^{\prime}}\right) \cdot \vec{r}_{n^{\prime} g}$. If in Eq. (3), the frequency denominator in the sum over $\left|n^{\prime}\right\rangle$ states could be approximated by a constant as independent of $n^{\prime}$, then from closure property we would have

$$
\alpha_{\text {chiral }} \propto \sum_{n^{\prime}} \vec{r}_{g n} \cdot\left(\vec{r}_{n n^{\prime}} \times \vec{r}_{n^{\prime} g}\right)=0 .
$$

Thus the nonvanishing $\alpha_{\text {chiral }}$ for SFG comes only because the spread of the frequency denominators is not negligible.

We have also attempted to measure SFG in transmission from a $2.5 \mathrm{M}$ water solution of arabinose. In order to benefit from resonant enhancement, we would like to have the sum frequency $(\omega)$ as close to an absorption peak as possible. Limited by our laser source, we were able to reach $\omega=50400 \mathrm{~cm}^{-1}(198.5 \mathrm{~nm})$ using $\omega_{1}=28200 \mathrm{~cm}^{-1}$ $(355 \mathrm{~nm})$ and $\omega_{2}=22200 \mathrm{~cm}^{-1}(450 \mathrm{~nm})$. It lies on the shoulder of the first absorption band of arabinose. In comparison, Rentzepis et al. had $\omega=43210 \mathrm{~cm}^{-1}$ [2] and Fischer et al. had $\omega=37594 \mathrm{~cm}^{-1}$ [4], both being far away from resonance. We had a detection limit of $\alpha_{\text {chiral }}=3 \times 10^{-42} \frac{\mathrm{m}^{4}}{\mathrm{~V}}$ at $\omega=50400 \mathrm{~cm}^{-1}$ and $\alpha_{\text {chiral }}=6 \times 10^{-43} \frac{\mathrm{m}^{4}}{\mathrm{~V}}$ at $\omega=46990 \mathrm{~cm}^{-1}$. Despite all the effort, we failed to observe SFG from the arabinose solution. Thus, for the frequencies we tried, $\alpha_{\text {chiral }}$ for arabinose is at least 10 times less than $\alpha_{\text {chiral }}=3.3 \times 10^{-41} \frac{\mathrm{m}^{4}}{\mathrm{~V}}$ of a BN molecule in THF measured with the SF beam at $322 \mathrm{~nm}$.

In summary, we have demonstrated that visible-visible SFG in chiral liquids is observable. The chiral nonlinear susceptibility is however small as it suffers from molecular orientational average. Delocalized electrons associated with the chiral molecular structure and resonant enhancement could be effective in bringing SFG above the detection limit.

This work was supported by the Director, Office of Energy Research, Office of Basic Energy Sciences, Material Science Division of the U.S. Department of Energy under Contract No. DE-AC03-76SF00098. S. H.H. acknowledges the support of the Korea Science and Engineering Foundation.

*Present address: Lucent Bell Labs, Murray Hill, NJ.

${ }^{\dagger}$ To whom correspondence should be addressed.

[1] J. A. Giordmaine, Phys. Rev. 138, A1599 (1965).

[2] P. M. Rentzepis, J. A. Giordmaine, and K. W. Wecht, Phys. Rev. Lett. 16, 792 (1966).

[3] A. P. Shkurinov, A. V. Dubrovskii, and N. I. Koroteev, Phys. Rev. Lett. 70, 1085 (1993).

[4] P. Fischer, D. S. Wiersma, R. Righini, B. Champagne, and A. D. Buckingham, Phys. Rev. Lett. 85, 4253 (2000).

[5] M. A. Belkin, T. A. Kulakov, K.-H. Ernst, L. Yan, and Y. R. Shen, Phys. Rev. Lett. 85, 4474 (2000).

[6] T. Petralli-Mallow, T. M. Wong, J. D. Byers, H. I. Yee, and J. M. Hicks, J. Phys. Chem. 97, 1383 (1993); J. D. Byers, H. I. Yee, T. Petralli-Mallow, and J. M. Hicks, Phys. Rev. B 49, 14643 (1994).

[7] Xing Wei, Seok-Cheol Hong, A. I. Lvovsky, Hermann Held, and Y. R. Shen, J. Phys. Chem. B 104, 3349 (2000).

[8] P. Guyot-Sionnest and Y. R. Shen, Phys. Rev. B 38, 7985 (1988).

[9] Y. R. Shen, The Principles of Nonlinear Optics (J. Wiley, New York, 1984), Chap. 2.

[10] Y. R. Shen, in Frontiers in Laser Spectroscopy, Proceedings of the International School of Physics "Enrico Fermi," Course CXX, edited by T. W. Hänsch and M. Inguscio (North-Holland, Amsterdam, 1994), p. 139.

[11] J. D. Byers and J. M. Hicks, Chem. Phys. Lett. 231, 216 (1994). 